\title{
Diastereoselective reactions of enolates
}

\author{
M. Braun*, H. Sacha, D. Galle, S. Baskaran \\ Institut für Organische Chemie, Universität Düsseldorf, D-40225 Düsseldorf, Germany
}

\begin{abstract}
Triphenylglycol-derived esters 2 and $8 \mathbf{a}$ have been applied in asymmetric aldol reactions. The homochiral propionates 7 and $\mathbf{8 a}, \mathbf{b}$ react with imines in a stereodivergent manner: Doubly deprotonated 7 delivers anti- $\beta$-lactams 14 whereas the lithium enolates of $8 \mathbf{a} / \mathbf{b}$ afford cis- $\beta$-lactams 15 in $87-97 \%$ e.e. Diastereoselective carbon silylation occurs when deprotonated $\mathbf{8 a}$ is treated with chlorotrimethylsilane. Approaches towards the construction of quaternary carbon centers are based on diastereoselective carboxalkylations of enolates with menthylchloroformate 17 and on a novel tandem reaction $(16 \rightarrow 20)$.
\end{abstract}

The generation of preformed enolates discovered three decades ago turned out to be extremely fruitful with respect to the formation of carbon-carbon bonds in a diastereoselective and/or enantioselective manner (1). Thus, the versatility of the aldol reaction has been enhanced substantially due to preformed enolates (2).

A couple of years ago, we reported on $(R)$ - and $(S)$-2-hydroxy-1,2,2-triphenylethyl acetate 2 ("HYTRA"), a chiral reagent which offers a solution for the long-standing problem of the stereoselective aldol addition of $\alpha$-unsubstituted enolates (3). HYTRA 2 is readily available from mandelic acid via triphenylglycol 1 . As shown in the illustrative procedure given below the crude mixture of adducts (3: predominant diastereomer) delivers $\beta$-hydroxycarboxylic acid 4 upon alkaline hydrolysis. Thereby, the chiral auxiliary reagent 1 is recovered and can be reused (4). The HYTRA-aldol method has been widely applied in syntheses of natural products and drugs (5).

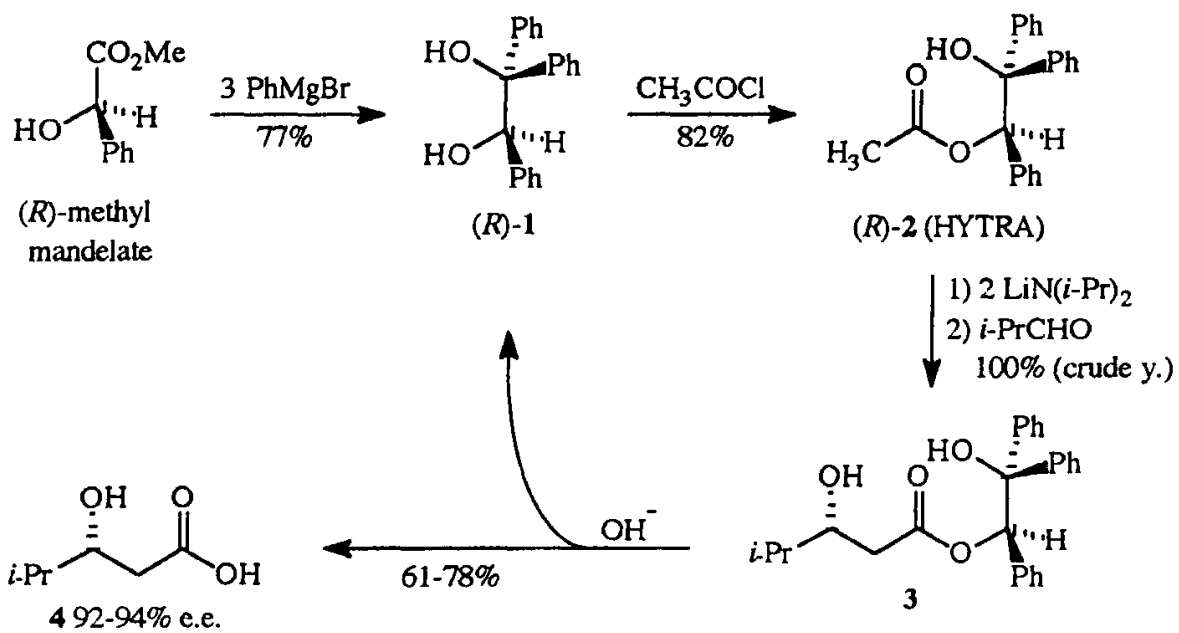

Whereas both enantiomers of syn-aldols 5 and ent-5 are available by several excellent procedures (2) the controlled and predictable preparation of anti-aldols 6 and ent-6 was a "problem child" of stereoselective synthesis, and solutions haven't been uncovered until very recently. Guided by the fact that triphenylglycol 1 is an extremely easily available reagent, we tried to apply triphenylglycol-derived propionates 7 in order to bring about anti-selective aldol additions which are diastereofacially selective as well.<smiles>[X]C(=O)[C@H]([R2])[C@@H]([R2])O</smiles><smiles>[X]C(=O)C([R4]#[SH])[C@@H]([R2])O</smiles><smiles>[X]C(=O)[C@H]([R])[C@@H]([R2])O</smiles><smiles>[X]C(=O)[C@H]([R2])[C@H](C)O</smiles> 
Thus, triphenylglycol 1 is esterified to give the propionate $7(96 \%)$ which is converterd into the $O$-silylprotected ester 8 by one pot deprotonation, silylation, and subsequent acidic hydrolysis. When 8 is deprotonated with lithium isopropylcyclohexylamide (LICA) transmetalated by the addition of dichlorocyclopentadienyl-zirconium, and finally treated with aldehydes, the predominant formation of anti-adducts 9a results. The anti:syn-ratios lie between $88: 12$ and $98: 2$. High diastereofacial selectivity is reached as well: diastereomeric ratios of $9 \mathrm{a}: \mathbf{9 b}$ range from $95: 5$ to $>98: 2$. Alkaline hydrolysis of the esters 9 provides carboxylic acids (e. g. 10, $\mathrm{R}=\mathrm{Ph}$ in $96 \%$ e.e.). On the other hand, reduction with $\mathrm{LiAlH}_{4}$ affords diols $11(\mathrm{R}=i$-Pr, $t$-Bu) in enantiomeric excesses of $>96 \%$ e.e. ( $6 \mathrm{a})$.

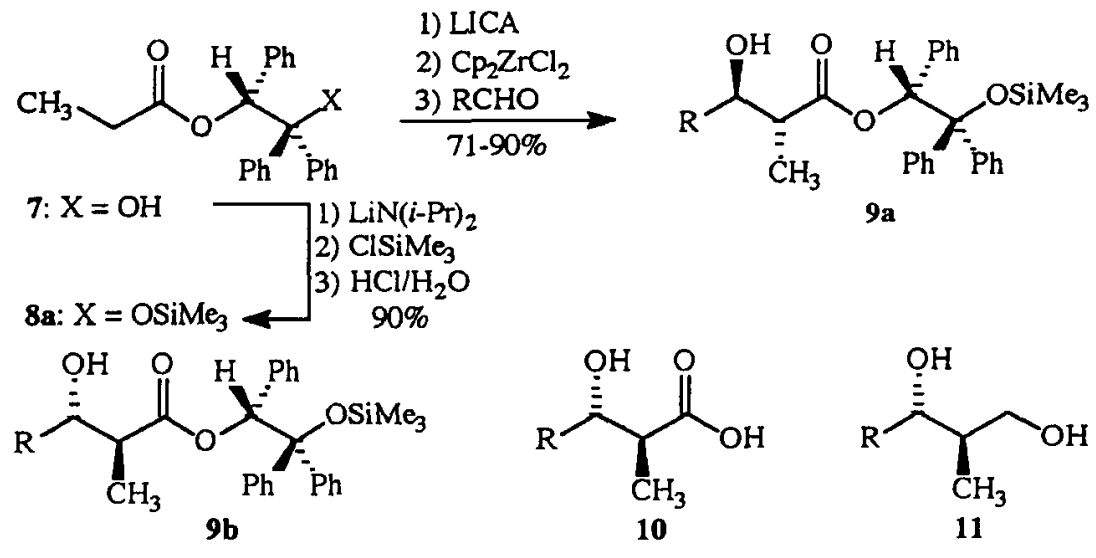

The X-ray structure analysis of 8 (6b), shown in Fig. 1, reveals a remarkably long carbon-carbon distance ( $1.56 \AA$ ) of the triphenylethane moiety (C4-C5). This is explained by the accumulation of sterically demanding substituents at vicinal carbon atoms, and one may assume that the bulkiness of the diphenyltrimethylsiloxy-methane unit plays an important role with respect to the stereoselecivity which is reached in aldol additions of the corresponding zirconium enolate.

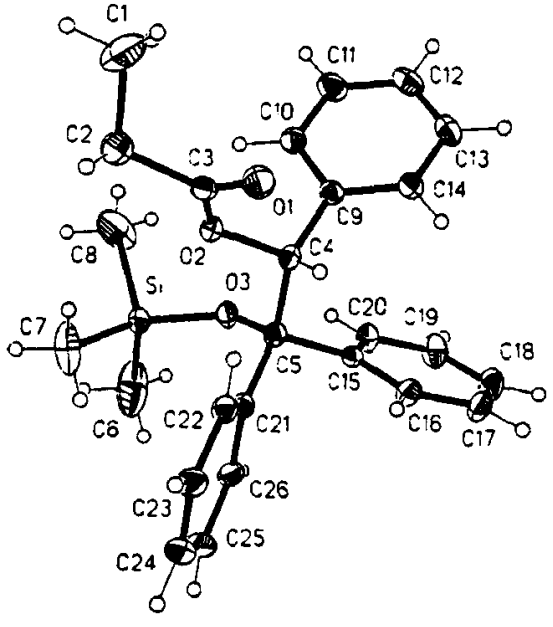

Fig. 1: X-Ray Structure of $8 \mathbf{a}$

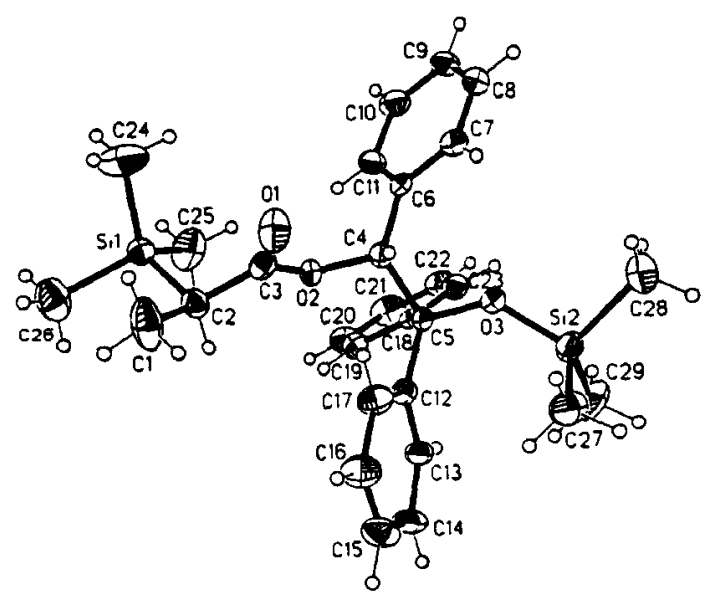

Fig. 2: X-Ray Structure of 13

The following unexpected reaction might be caused by the sterical demand of the alcoholic moiety of the ester 8a: When the propionate $8 \mathbf{a}$ is deprotonated and the enolate formed thereby is treated with chlorotrimethylsilane, the formation of the silylketene acetal 12 has been anticipated. However, the $\alpha$-carbon atom, obviously the most accessible nucleophilic center of the enolate, is silylated rather than the oxygen atom so that the $\alpha$-silyl ester 13 is obtained. This carbon silylation reaction occurs with remarkable diastereoselectivity (diastereomeric ratio: $94.5: 5.5$ ). The configuration of the major diasteromer 13 is proven by the $X$-ray structure analysis shown in Fig. 2 (6b). 
<smiles>CO[C@H](OC(c1ccccc1)[C@H](OC(C)(C)C)c1ccccc1)c1ccccc1</smiles>

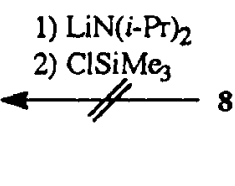

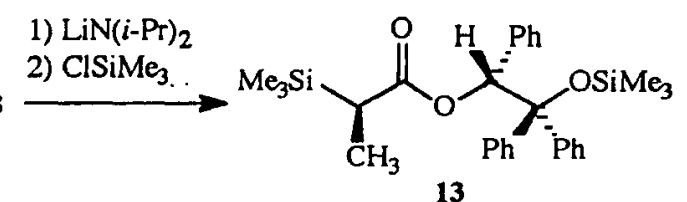

The esters 7 and $8 \mathbf{a}, \mathrm{b}$, although homochiral, give rise to a different stereochemical outcome in the reaction with $\mathrm{N}$-protected imines which leads to the formation of $\beta$-lactams. Thus, trans- $\beta$-lactams 14 are obtained in the condensation of imines with doubly deprotonated ester 7 whereas cis-azetidinones 15 are formed predominantly when the enolates of propionates $8 \mathrm{a} / \mathrm{b}$ are allowed to react with imines. The chiral auxiliary group is cleaved in situ. Remarkable enantioselectivities of both trans and cis- $\beta$-lactams are reached (Table 1).<smiles>CCC(=O)OC(c1ccccc1)C(O)(c1ccccc1)c1ccccc1</smiles>

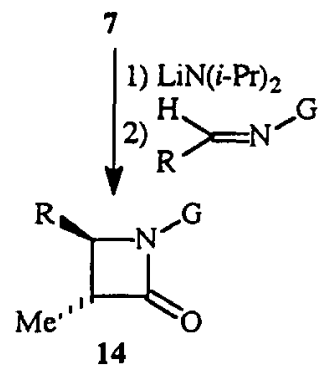<smiles></smiles><smiles>[X]C(c1ccccc1)(c1ccccc1)[C@H](OC(=O)CC)c1ccccc1</smiles>

8a: $X=\mathrm{OSiMe}_{3} ; 8 \mathrm{~b}: \mathrm{X}=\mathrm{OMe}$

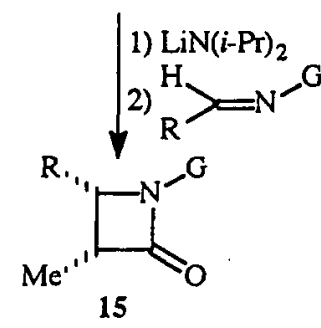

Table 1. $\beta$-Lactams 14 and 15 by condensation of esters 7 and $8 \mathrm{a}, \mathrm{b}$ with imines

\begin{tabular}{c|c|c|c|c}
\hline $\mathrm{R}$ & Ester & trans-14:cis-15 & c.e. & Yield \\
\hline Phenyl & 7 & $94: 6$ & $>97 \%$ & 83 \\
2-Furyl & 7 & $96.5: 3.5$ & $>97 \%$ & 85 \\
\hline Phenyl & $\mathbf{8 a}$ & $3.5: 96.5$ & $87 \%$ & 82 \\
2-Furyl & $\mathbf{8 b}$ & $9: 91$ & $>97 \%$ & 87 \\
\hline
\end{tabular}

Another strategy for the stereoselective formation of carbon-carbon bonds relies on the reaction of prochiral enolates with chiral electrophiles. This concept has been used by us for the generation of stereogenic quaternary carbon centers, when we became interested in studies directed towards a synthesis of Fredericamycin A (7). To give an example, doubly deprotonated indanecarboxylic acid 16 reacts in a diastereoselective carboxalkylation reaction to give predominantly the acid $18 \mathrm{a}$ when treated with the commercially available chloroformate 17 derived from (-)-menthol. The diasteromeric ratio of $18 \mathrm{a}: \mathbf{1 8 b}$ amounts $9: 1$. However, higher diastereoselectivities can be obtained when either ester or ketone enolates are used or when the chiral reagent $\mathbf{1 7}$ is substituted by the corresponding chloroformate derived from 8-phenylmenthol (8).

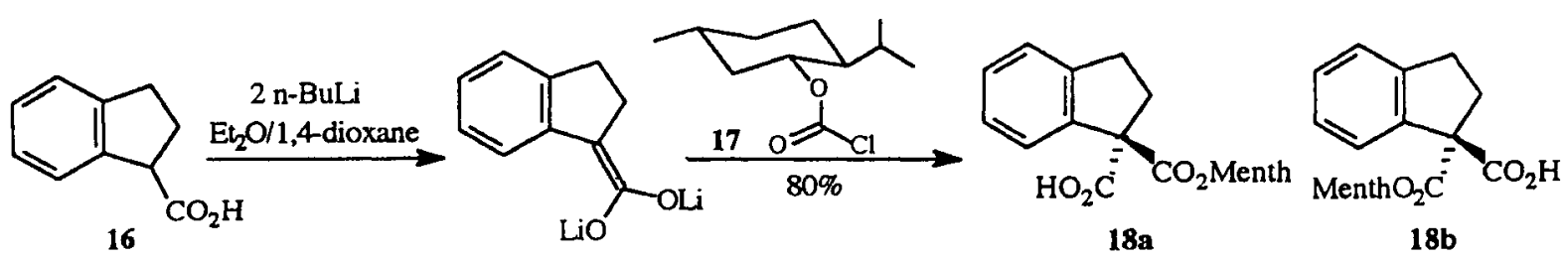


In another approch for the synthesis of the core skeleton 21 of Fredericamycin A, a surprizing tandem reaction has been found: When the dianion of 16 is treated with ethoxyphthalide 19 the spirocompound 20 forms diastereoselectively in one pot. Subsequent oxidation affords the diketone 21.<smiles>O=C(O)C1CCc2ccccc21</smiles>

16<smiles>CCOC1OC(=O)c2ccccc21</smiles><smiles>O=C1c2ccccc2[C@@H](O)[C@]12Cc1ccccc12</smiles>

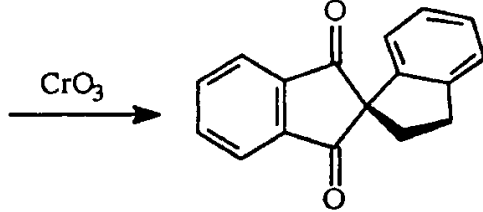

21

A rationale for the formation of $\mathbf{2 0}$ is given by a tandem reaction which consists of a Claisen, decarboxylation and aldol sequence.

\section{References}

(1) C. H. Heathcock in Modern Synthetic Methods 1992 (Ed.; R. Scheffold), Verlag Helvetica Chimica Acta, Basel; VCH, Weinheim 1992, p. 1.

(2) D. A. Evans, J. V. Nelson, T. R. Taber, Top. Stereochem. 13, 1 (1982); C. H. Heathcock in Comprehensive Organic Syntheses (Ed.: B. M. Trost), Pergamon Press, Oxford, 1993, vol. 2, chapter 1.6; M. Braun in Advances in Carbanion Chemistry (Ed.: V. Snieckus), JAI Press, Greenwich, CT, USA 1992, vol. 1, p. 177.

(3) M. Braun, Angew. Chem. Int. Ed. Engl. 26, 24 (1987).

(4) M. Braun, S. Gräf, S. Herzog, Org. Synth. 72, 32 (1993); M. Braun, S. Gräf, Org. Synth. 72, 38 (1993).

(5) M. Braun, H. Sacha, J. Prakt. Chem. 335, 653 (1993); and references given therein.

(6) (a) M. Braun, H. Sacha, Angew. Chem. Int. Ed. Engl. 30, 1318 (1991). (b) H. Sacha, D. Waldmuller, M. Braun, Chem. Ber. 1271959 (1994).

(7) R. Misra, R. C. Pandey, B. D. Hilton, R. P. Roller, V. Silverton, J. Antibiot. 40, 786 (1987).

(8) W. Trypke, A. Steigel, M. Braun, Synlett 1992, 827. 